\title{
Ein Beitrag zur Frage des cerebralen Fiebers.1)
}

\section{Von}

\author{
0. Fischer (Prag).
}

Mit 1 Textfigur.

(Eingegangen am 10. April 1912.)

Der Sachs-Aronsohnsche Wärmestich hat den sicheren Beweis für den Zusammenhang von Hirnläsion und Temperatursteigerung erbracht; damit war auch für die bereits früher wiederholt geäußerte Ansicht, daß die nach manchen Hirnerkrankungen auftretenden Temperatursteigerungen als ,cerebrales Fieber" anzusehen sind, eine experimentelle Grundlage gegeben. Temperatursteigerungen dieser Art kommen bej verschiedenen Erkrankungen des Gehirns vor; so findet man manchmal in Fällen von ausgedehnter Hämorrhagie des Großhirns, besonders wenn die Basalganglien mit ergriffen werden, Temperatursteigerungen, die auf eine andere Weise nicht zu erklären sind; etwas Ähnliches gilt auch für Tumoren. Die Temperatursteigerung, die sich sehr häufig nach den Krampfanfällen der Epileptiker oder Paralytiker einstellt, ist mit größter Wahrscheinlichkeit auch derart aufzufassen, obzwar die Möglichkeit nicht von der Hand zu weisen ist, daß diese Temperatursteigerung toxisch bedingt wäre, zurückführbar auf das Toxin, welches eventuell den Anfall selbst provoziert hatte; in diesem Fall würden dann Anfälle und Temperatursteigerung nicht in direktem ätiologischen Zusammenhang sein, sondern wären nur koordiniert. Auch das Fieber bei Delirium tremens könnte man als cerebrales Fieber erklären, obzwar hier ebenfalls derselbe Einwand wie bei den Krampfanfällen erhoben werden kann. Weiter hat man gefunden, daß Phthisiker nicht nur auf Tuberkulininjektionen mit Temperatursteigerung reagieren, sondern auch auf Injektion von Kochsalzlösung, ja auch dann, wenn man nur pro forma, ohne etwas zu injizieren, eingestochen hat. Eine derartige Temperatursteigerung kann natürlich gar nicht anders als auf suggestivem Wege, also auf rein nervöser Basis, entstanden sein. Dasselbe gilt auch vom hysterischen Fieber, das, obzwar von vielen Autoren negiert, dennoch als sicher bestehend angesehen werden kann, das heißt es gibt Fälle von Hysterie, bei denen sich zeitweise sehr hohes

1) Vortrag, gehalten in der „Wissenschaftlichen Gesellschaft deutscher Ärzte in Böhmen" in Prag am 22. März 1912. 
Fieber einstellt, das sich Medikamenten gegenüber sehr refraktär verhält, auf sonstige somatische Veränderungen nicht zurückführbar ist und ohne wesentliche Alteration des körperlichen Wohlbefindens oft längere Zeit anhält. Unter Umständen lassen sich hierbei auch suggestive Einflüsse geltend machen.

Nach all dem existiert auch beim Menschen eine cerebral verursachte Temperatursteigerung; bei der Frage aber, wie häufig dies vorkommt, respektive wann wir eine Fiebertemperatur als cerebral verursacht anzusehen haben, kommen wir auf sehr unsicheren Boden. Denn das bisher bekannte einschlägige Material ist sehr gering und noch dazu zum Teil von so zweifelhafter Wertigkeit, daß für die Feststellung der näheren Bedingungen dieses „,cerebralen Fiebers“ noch eine große und recht genau untersuchte Kasuistik notwendig sein wird.

Deswegen ist eine jede einzelne Beobachtung, die zu dieser Frage in Beziehung steht, von Wichtigkeit, und aus diesem Grunde soll auch folgender Fall mitgeteilt werden, um so mehr er sehr einfache, leicht übersehbare und in mancher Hinsicht an ein Experiment erinneInde Verhältnisse bietet. -

45 jährige Beamtenfrau, die am 9. Aug. 1911 in meine Beobachtung kam (Sanatorium Weleslawin bei Prag). Sie soll früher immer gesund gewesen sein; hysterischer Charakter; seit 1/2 Jahr zeigten sich Unregelmäßigkeiten der Periode, sie wurde zerstreut, fahrig, manchmal traurig und äußerte wiederholt nicht recht motivierte Suicidideen. Vor 2 Monaten bekam sie plötzlich einen „Tobsuchtsanfall", der sich bald legte, sie blieb aber ängstlich; klagte manchmal über optische Halluzinationen ängstlichen Inhalts (sah Teufel).

Eingebracht zeigte die Kranke das Bild ängstlicher Ratlosigkeit mit ausgesprochenem Negativismus; sie war hochgradig abgemagert, sonst somatisch nichts Besonderes.

In den ersten Tagen blieb der Zustand im allgemeinen unverändert; aber auch in der ängstlichen Erregung hatte das Gebaren der Kranken einen theatralischen Anstrich. Abstinenz und Sondenfütterung.

Am 14. Aug. entwickelte sich ein negativistischer Stupor; die Kranke lag regungslos ausgestreckt im Bette, die gesamte Körpermuskulatur war leicht tonisch gespannt, die Hände fest zur Faust eingekrallt, der Gesichtsausdruck meist stumpf, andeutungsweise aber auch ängstlich-delirant. Dabei war aber trotzdem der Negativismus nicht geschwunden, denn bei jedem Versuche einer passiven Bewegung spannte die Kranke die ergriffene Extremität auf das intensivste im Sinne des Widerstrebens an. Erst nach Aufhören dieses Versuches stellte sich wieder der frühere Muskeltonus ein.

Am 15. Aug. trat eine Temperatursteigerung auf, die im weiteren Verlaufe beinahe den Typus einer Febris continua hatte. Antipyretica hatten, wie die Tabelle zeigt, eigentlich keinen EinfluB; nur Aspirin in $1 \mathrm{~g}$-Dosen setzte die Temperatur vorübergehend herab, dagegen wirkten Migränin ( $\mathrm{g}$ ), Antipyrin (l g), Chinin $(1 / 2 \mathrm{~g})$ überhaupt nicht. In dieser Phase war ein ganz profuser, ständiger Schweißausbruch das auffälligste. Bei Tag und Nacht, ganz ohne Zusammenhang mit den täglichen Temperaturschwankungen, und auch ganz ohne Zusammenhang mit den eingegebenen Medikamenten schwitzte die Kranke ständig so, daB der Schweiß förmlich vom Körper herunterrann; kaum hatte man die Haut der 
Kranken abgewischt, war auch schon der Schweiß in Form kleinster Tröpfchen auf der Oberfläche sichtbar; die Schweißproduktion war so intensiv, daß die Bettwäsche der Kranken davon ständig feucht war.

Eine somatische Ursache für diese merkwürdige Temperatursteigerung war nicht zu finden; die inneren Organe waren gesund, der Augenhintergrund war normal; es ließ sich zwar eine alte Otitis media suppurativa nachweisen, die aber keinerlei Exacerbation zeigte und deswegen ätiologisch ausgeschlossen werden konnte. Eine zweimal angestellte Fickersche Typhusprobe fiel negativ aus. Der Harn war normal. Am 22. Aug. entstand auf Grund der ganz steifen Haltung

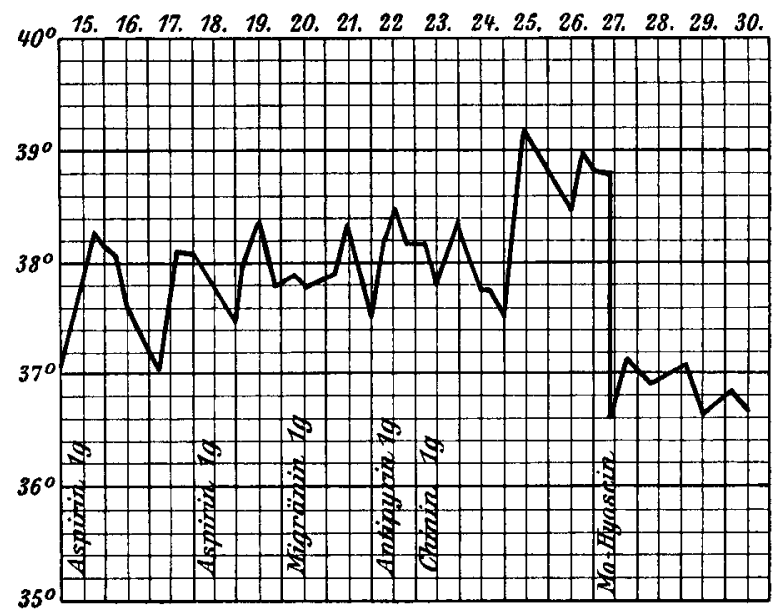

Fig. 1.

der Kranken an einer Ferse eine Druckblase und am Sacrum ein ganz oberflächlicher Decubitus; beide wurden bakteriologisch untersucht und konnten auch in keiner Weise für das Fieber ätiologisch in Betracht kommen. Eine Lumbalpunktion am 26. Aug. ergab vollkommen normale Flüssigkeit. Im Blute fanden sich dagegen 16000 weiße Blutkörperchen. Darauf wurde eine genaue Untersuchung des Abdomens beschlossen, wozu der Kranken am 27. Aug. zwecks entsprechender Entspannung der Bauchdecken eine geringe Dosis von Hyoscin und Morphium injiziert wurden; da eben nur eine Entspannung erzielt werden sollte, wurden nur ganz geringe Mengen angewendet, und zwar $0,3 \mathrm{mg}$ Hyoscin und $8 \mathrm{mg}$ Morphium. Etwa nach 10 Minuten trat unter kollapsartigem Erblassen ein plötzlicher Temperatursturz auf $36,6^{\circ}$ ein, wobei merkwürdigerweise die Schweißsekretion, entgegen dem Verhalten bei einem gewöhnlichen Kollaps, genau so plötzlich versiegte. Die Untersuchung des Abdomens ergab keine nachweisbare Veränderung der Bauchorgane.

Von dem Zeitpunkt an blieb nun die Kranke vollkommen fieberfrei, trotzdem sich sonst der Zustand in keiner Weise verändert hatte: der Stupor blieb im gleichen, ebenso auch die Spasmen; die hochgradige Schweißsekretion war verschwunden und auch die Blutleukocytose sank auf 7000 .

Der weitere Verlauf gestaltete sich folgendermaßen: Ende September ließ der Stupor etwas nach, die Kranke wurde allmählich mehr und mehr zugänglich, und schließlich war sie so weit gebessert, daß sie zwar noch ein wenig gehemmt, doch schon am gesellschaftlichen Leben in der Anstalt teilnehmen konnte. Mitte 
Oktober stellte sich eine heftige Menstrualblutung ein, die durch 10 Tage andauerte. Damit setzte auch der frühere Stupor in ganz gleicher Art und Stärke ein; zu einer Temperatursteigerung kam es dabei aber nicht. Anfang Dezember ließ der Stupor allmählich nach, dabei bot die Kranke das Bild einer Katatonie dar; den Ärzten gegenüber war sie negativistisch, wogegen sie mit dem Wartepersonal oft recht viel sprach, war sehr kindisch, zeigte allerlei Maniriertheiten, beim Anund Ausziehen war sie meist ausgesprochen negativistisch, manchmal auch beim Essen und Waschen; ein Brief von ihrem Mann brachte sie immer in kindische Ekstase; sie sprach dann ständig und tagelang immer ein und dasselbe vor sich hin, wie z. B. : ,"Ein Brief von meinem Mann“ . . . oder: ,Mein Mann wird kommen.“

Allmählich besserte sich der Zustand immer mehr; Anfang Januar kam es wieder zu einer protrahierten Menstrualblutung, aber diesmal ohne Alteration des psychischen Zustandes. Gegen Ende Januar zeigte sich eine leichte manische Erregung, die im Verlaufe von 14 Tagen verschwand. Die Genesung scheint eine vollkommene gewesen zu sein; die Kranke war geistig in jeder Hinsicht normal, zeigte insbesondere keinerlei Intelligenzdefekt, war für den Stupor amnestisch, und wußte sich eigentlich nur bruchstückweise an einzelne Geschehnisse aus der depressiven Initialphase zu erinnern.

Somatisch hatte sich Pat. sehr erholt, das Gewicht war von 49 auf $59 \mathrm{~kg}$ gestiegen; die Corneal- und Pharynxreflexe fehlten, sonstige Stigmen waren aber nicht nachweisbar. Die Kranke wurde nach Hause entlassen und blieb bis zum heutigen Tage gesund.

Mit Sicherheit wird sich der geschilderte Fall kaum in unser bisheriges System der Psychosen einreihen lassen, so daß man die Einteilung dieser Psychose mehr dem Geschmack eines jeden einzelnen überlassen muß; darin werden sich aber wohl alle einig bleiben, daß hier das Klimakterium als ein wichtiger ätiologischer Faktor anzusehen ist. Denn die Psychose ist nicht nur in der Zeit des Klimakteriums eingetreten, sondern ist nach wesentlicher Besserung wieder nach Einsetzen einer neuerlichen Menstrualblutung in der früheren Stärke rezidiviert. Die Krankheitsform dürfte, wie gesagt, vielleicht schwer einzureihen sein; denn einerseits waren hier depressive Züge vorhanden - die Genesung erfolgte sogar erst nach einer kurzen manischen Phase -, so daß man an ein manisch-depressives Irresein denken konnte, anderseits erinnert gar manches an eine Katatonie: so der schwere Stupor, das maniriert-katatone Wesen, das kindische Benehmen, und schließlich könnte man auch auf das theatralische Wesen und die fehlenden Schleimhautreflexe hinweisen und dadurch auch eine eventuelle Zusammengehörigkeit mit der Hysterie in Erwägung ziehen.

Doch die Klassifikationsfrage können wir hier einstweilen beiseite lassen. Viel interessanter und wichtiger erscheint uns das Verhalten der Körpertemperatur, indem sich hier im Verlaufe eines schweren negativistischen Stupors eine Temperatusteigerung mit dem Charakter einer Febris continua einstellte, die sich durch Antipyretica kaum beeinflussen ließ; von den angewendeten Antipyreticis, als Chinin, Migränin, Antipyrin und Aspirin, hatte nur das letzte eine vorübergehende Temperatursenkung zur Folge; deswegen erscheint um so merkwürdiger die 
prompt entfiebernde Wirkung der in ihrer Dosis doch so minimalen Hyoscin-Morphium-Injektion. Es würde vielleicht nicht wundernehmen, wenn nach dieser Injektion - sowie nach dem Aspirin - die Temperatur gesunken und wieder zur ursprünglichen Höhe gelangt wäre, denn von Hyoscin wie auch von Morphiumist es ja bekannt, daß es häufig die Temperatur herabsetzt; das Auffallende liegt aber in der dauernden Entfieberung, so daß man sich bei diesem eigenartigen Zusammentreffen nichts anderes denken kann, als daß durch die Injektion auch die eigentliche Ursache der Temperatursteigerung beseitigt worden ist. Es muß betont werden, daß es nicht die mukellähmenden Komponenten der Injektion waren, die hier wirkten; denn als nach einigen Stunden die motorische Erschlaffung vorübergegangen war, verfiel die Kranke in denselben Muskeltonus, in dieselbe steife katatone Haltung, aber die Temperatursteigerung und die abnorme Schweißabsonderung blieben dennoch aus. Das refraktäre Verhalten der Kranken gegenüber den Antipyreticis und die überaus starke Hyperhydrose hatten mich schon im Anfang des Fiebers zu der Ansicht geführt, daß wir vielleicht eine Art von „,erebralem Fieber" vor uns haben; der prompte (aber in keiner Weise erwartete) Erfolg der Hyoscin-Morphium-Injektion ließ nun kaum eine andere Deutung zu: Nach dem ganzen Sachverhalt wird man ein einfaches ",posthoc und nicht propter hoc" abweisen müssen; eine infektiöse Ursache war nicht vorhanden, hätte auch nicht durch die Injektion so prompt beseitigt werden können; wenn auch starke Muskelanstrengungen Temperaturerhöhung hervorzurufen imstande sind, so können sie so hohe Temperaturen, wie sie unser Fall zeigte, doch nicht bewirken; schließlich verblieb auch nach der Injektion die Muskelspannung, und nur die Temperatursteigerung mit der Hyperhydrose war geschwunden. Nun kann man sich einen so schweren Stupor doch nur als Folge einer schwereren Alteration des Gehirns entstanden denken (wofür wir ja bereits anatomische Beweise haben); bei einer so schweren Alteration des ganzen Gehirns können unter Umständen auch die „Zentren“, welche die Temperaturverhältnisse des Körpers regulieren. - und solche sind durch das Tierexperiment unbedingt bewiesen - erkranken und dann müßte man auch eine Veränderung in der Wärmeregulierung erwarten; nach Analogie des Tierexperimentes müßte bei einem Reiz auf das ,Zentrum“ eine erhöhte Körpertemperatur resultieren; Hyoscin als auch Morphium (welches von den zwei Alkaloiden in unserem Fall das wirksame war oder ob es beide waren, läßt sich natürlich jetzt nicht entscheiden) sind nun Gifte, welche auf nervöse Elemente lähmend einwirken; sie sind also imstande, einen etwa vorhandenen abnormen Reiz im Wärmezentrum zu beseitigen und dadurch die abnorme Fiebertemperatur wieder in normale Grenzen zu bringen, das heißt sie wirken in einem solchen Falle prompt entfiebernd. 
In gleicher Weise kann man sich auch die gleichzeitige Beseitigung der Hyperhydrose erklären, die wohl in ganz ähnlichen Vorgängen wie die Hyperthermie ihre Ursache hat. Ja wir könnten in unseren Schlußfolgerungen noch einen Schritt weiter gehen: Von beiden Alkaloiden weiß man nämlich, daß sie in geringen Dosen zuerst die Hirnrinde angreifen und erst in größeren Dosen die subcorticalen Zentren; daraus daß in unserem Falle nur so minimale Mengen die geschilderte Wirkung hatten, sollte man schließen, daß das in Frage kommende Zentrum in der Hirnrinde gelegen wäre. Ein Beweis für diesen Schluß läßt sich gegenwärtig nicht erbringen; doch fehlt es nicht an Behauptungen in der Literatur, daß Temperaturveränderungen mit Hirnrindenveränderungen in Beziehung stehen.

Es läßt sich weiter auch nicht unterscheiden, ob wir in unserem Falle nur eine Art von Hyperthermie vor uns haben oder ob wir diesen Zustand auch als wirkliches Fieber auffassen sollen; ausschlaggebend dafür wäre der Nachweis eines veränderten Stoffwechsels, was aber aus selbstverständlichen Gründen nicht festgestellt wurde; es konnte nur sichergestellt werden, daß der Harn keine abnormen Bestandteile enthält.

Auf einen Umstand möchte ich noch hinweisen, der dafür zu sprechen scheint, daß der Stoffwechsel doch nicht so ganz intakt gewesen sein kann, nämlich auf die nachgewiesene Blutleukocytose zur Zeit der Hyperthermie; dieser Befund ist um so interessanter, als ähnliches auch bei den Wärmestichtieren beobachtet wurde (Löwy und Richter).

Einen ähnlichen Fall konnte ich in der Literatur nicht finden; und doch scheint es mir nicht ausgeschlossen, daß solche Verhältnisse häufiger vorkommen; denn ich weiß mich aus eigener Erfahrung mancher rätselhafter Fieberzustände bei Psychosen zu erinnern, die man gar nicht recht einzureihen wußte und für die man auch keine Ursache finden konnte; vielleicht hätte man in solchen Fällen im Morphium-Hyoscin ein Mittel zur Diagnose, ev. auch zur Beseitigung dieser Zustände, fa es würden sich dann vielleicht noch manche klinische Eigenarten dieser Zustände aufdecken lassen. Doch immerhin muß man sich vor Augen halten, daß diese Erklärung noch auf hypothetischer Basis beruht; denn bei den so komplizierten Verhältnissen im Organismus kann es unschwer zu schier unabsehbaren Zufällen kommen, so daß auch das Wahrscheinlichste nur zu leicht zu einem groben Irrtum wird. Aber auch über so exzeptionelle Fälle, wie der gerade geschilderte, darf man nicht einfach zur Tagesordnung übergehen, sie sollen zum Nachdenken anregen und für weitere Beobachtungen den Weg weisen. 